\title{
STELLAR MODELS OF $\varepsilon$ ERIDANI CONSTRAINED BY STELLAR SEISMOLOGY
}

\author{
D. B. Guenther \\ Center for Solar and Space Research \\ Yale Department of Astronomy \\ New Haven, CT 06511 \\ USA
}

\begin{abstract}
Because Epsilon Eridani $(\mathrm{Z}=0.013)$ appears about $0.2 \mathrm{mag}$ above the $\mathrm{Z}=$ 0.02 zero-age main sequence, zero-age models of Epsilon Eridani must be evolved off the ZAMS to ages much older than its rapid rotation and high chromospheric activity suggests. To resolve this conflict we propose that either Epsilon Eridani is part of a binary system or that its metal content has been underestimated. To test these hypotheses we use not only the stellar constraints of luminosity and effective temperature but also impose that the characteristic frequency spacing of the oscillation spectra of the models match the value observed by Noyes et al. (1984).
\end{abstract}

\section{DISCUSSION}

Previous work (Guenther and Demarque 1986) has shown that in order to produce stellar models of Epsilon Eridani which satisfy all the observational constraints (surface temperature, luminosity, implied age, atmospheric abundances, and characteristic p-mode frequency spacing) it is necessary to use a mixing length parameter which is much lower than that used to produce the standard solar model. Not satisfied with this ad hoc assumption we have tried to find more justifiable solutions to the problem.

The high luminosity of Epsilon Eridani could be accounted for if it were part of a binary system. We could not justify this assumption. If it is part of a binary star system than either the orbit is nearly face on (a low probability assumption) or its companion has a very small mass. The former case is implied by the null results of speckle interferometric observations (McAlister 1978, and Hartkopf and McAlister 1983) and radial velocity measurements (Beavers and Eitter 1986). Even if the latter case were true the low luminosity of the small companion would not help explain the star's high luminosity. The metal content, $[\mathrm{Fe} \mathrm{I} / \mathrm{H}]=-0.19(\mathrm{Z}=0.013)$, and effective temperature, $\log \left(\mathrm{T}_{\mathrm{eff}} / \mathrm{K}\right)=3.70$, of Epsilon Eridani have been determined from model atmosphere calculations (Oinas 1974). The lower than solar Z suggests that Epsilon Eridani is older than the Sun's $4.7 \mathrm{Gyr}$. This is at odds with the young age implied by its high chromospheric activity and rapid rotation rate. We believe the metal abundance has been underestimated because of the presence of bright plage regions on the surface of the star. The higher temperatures of these regions effectively fills in the metal lines with respect to the continuum. LaBonte and Rose (1985) have shown that this effect occurs in the plage regions of the sun. When we assume a higher metal abundance it is relatively easy to 
produce models which satisfy all the observable constraints, including the p-mode characteristic frequency.

\section{CONCLUSIONS}

In this work the p-mode spectrum took a secondary role in tuning in on Epsilon Eridani's structure. It is important to know the star's distance, luminosity, surface temperature and composition well enough so that initial guess models can be constructed for use in a stellar seismological analysis. Using all available observation data we are lead to the conclusion that Epsilon Eridani is a young star, probalbly not part of an equalcomponent binary system, with solar to slightly metal rich metal abundance.

\section{REFERENCES}

Beavers, W. I. and Eitter, J. J. 1986, Ap. J. Suppl., in press.

Guenther, D. B. and Demarque, P. 1986, Ap. J., 301, 207.

Hartkopf, W. I. and McAlister, H. A. 1984, P.A.S.P., 96, 105.

LaBonte, G. J. and Rose, J. A., 1985, P.A.S.P., 97, 790.

McAlister, H. A. 1978. P.A.S.P., 90, 288.

Noyes, R. W., Baliunas, S. L., Belserene, E., Duncan, D. K., Horne, J., and Widrow, L. 1984, Ap. J. (Letters), 285, L23.

Oinas, V. 1974, Ap. J. Suppl., 27, 405. 